\title{
Refining models of nuclear matter
}

\section{The general expectation that the structure of the dense substance of a neutron star would be surprising has been more than outdone by speculations, but the nuclear data are not yet good enough to tell just what happens.}

WHAT exactly is nuclear matter, and where is it to be found? Since the discovery of pulsating stars more than two decades ago, and their identification (by Thomas Gold, see Nature 218,731; 1968) as stars made primarily from nuclei, electrons and neutrons, the answer to the second question has been taken for granted. But there is a difficulty with the natural view that the interior of a neutron star is simply a collection of neutrons moving about much as if they were inert atoms in a liquid.

If the force holding together a neutron star, believed to be the core of an exploded supernova, is simple gravity, it is difficult to understand how it can be massive enough to hang together against the obvious transition back to hydrogen atoms (for neutrons decay into electrons and protons, on the average in about 20 minutes if admittedly more slowly in strong gravitional fields).

Of course, the core of an exploded supernova contains not just neutrons but nuclei, not least those of the lighter elements involved in stellar nucleosynthesis, so that the interior of a neutron star may be an arrangement of relatively slowly moving nuclei in a sea of neutrons with a uniformly distributed electron gas as well. But then, if the pressure on the core of the star were increased, as it would be if the mass of the star were greater, the nuclei would be disrupted into protons and neutrons. And what if the central pressure were greater still? The constituents of the star would be the quarks of which nucleons are made. What forces would hold matter of that kind together?

What more natural than that the strong nuclear forces are responsible? If that is so, the interior of a neutron star resembles the interior of a nucleus. In other words, the interior of a neutron star is a collection of quarks held together by the gluons that constitute the nuclear cement - or a neutron star is a giant nucleus. That accords with observations (so far as they are complete) of the physical properties of neutron stars.

The density of a neutron star, for example, is more like that of a nucleus than of a molecular liquid. The density of nuclear matter must differ from that of the densest ordinary liquid by a factor of the order of $10^{12}$, the ratio of the volume of an atom and of a nucleus. One of the earliest attempts to calculate the properties of nuclear matter in this form (then called "quark matter") was due to George Chapline and Michael Nauenberg (Nature 264, 235; 1976). Starting from the position that the quarks must be

NATURE · VOL 362 · 1 APRIL 1993 relativistic, they concluded (from General Relativity) that the pressure of the fluid must be roughly a third of the energydensity of the nuclear matter.

But if this is what the interior of a neutron star is like, still more remarkable things are possible. One comes about because quarks themselves are not necessarily stable, but may decay from one into another radioactively by the release of an electron or heavier fermion. And the inverse decays are also possible. So it is reasonable to ask what mixture of quarks and electrons would be the most stable. There is a possibility that nuclear matter made of neutrons may partly consist of electrons, electrically balanced by an array of charged quarks which, if put together into separate nucleons, would make some neutrons and some different number of protons. That is not a surprising possibility, but a likely one. The question is not whether it happens, but under what circumstances, and where.

Even as recently as 1978 , it had not been established that the breakdown of neutrons into quarks would happen inside a neutron star. W.F. Fechner and P.C. Joss in that year made a judicious attempt to estimate the likelihood of this phase transition, which is what it would be. Their conclusion was that the existence of stable quark stars was "possible", and that they might well have properties not very different from those inferred from observation and from earlier calculations (Nature 274, 347; 1978).

But now, imaginations have moved further on. For one thing, there is a string of speculative calculations concerned with the effects of nuclear processes within such a star that would liberate neutrinos, then each presumed to have a chance of escaping from the star and carrying away energy. The effect of these processes would thus be to cool a newly formed neutron star with a pulse of neutrinos, perhaps like that observed on the Earth from the Magellanic supernova explosion early in 1987.

The supernova 1987a may be an observational proof of the reality of quark matter, for one of the processes by which neutrinos are emitted consists of the interaction of an up quark (one of the three quark components of a neutron) with a negative electron, leaving a strange quark and a neutrino. So there is a bias towards the formation of strange quarks, whence the term "strange matter" appropriately describing bulk objects consisting predominantly of strange quarks. (The term "strangelets" is used for droplets of the stuff.) The possibil- ity has even been raised that neutron stars of all forms may be metastable against conversion into strange matter.

Strange matter might even have practical value. Not so long ago, G.L. Shaw, M. Shin, R.M. Dalitz and M. Desai suggested making nuggets of strange matter (with mass anywhere between a very small fraction of a gram and many billions of tonnes). Then, they argued (Nature 337, 436; 1989), it would be possible to exploit the reactions by which the quark components of a neutron are converted into strange quarks to generate thermonuclear energy cheaply; just fire slow neutrons at a nugget of strange matter, collect the energy of the neutrinos emitted in some way or another and, in the process, watch the nugget of strange matter grow. A "thermonuclear breeder" is the appropriate term for that.

More recently, people have been brooding about the ways in which ordinary matter would progressively be converted into quark matter or even strange matter. In the form in which nuclei remain intact, the first thing that happens as the density of pressure in increased is that the charge distribution within nuclei is inverted; the energy involved in Coulomb repulsion increases less quickly than the surface energy. Then nuclear matter begins to form, at first as droplets. But then the droplets grow and become rod-like and plate-like ("spaghetti" and "lasagna" are the technical terms).

Earlier this year, C.P. Lorenz, D.G. Ravenhall and J. Pethick from the University of Illinois at Urbana more accurately calculated the equation of state by which the properties of these structures are determined (Phys. Rev. Lett. 70, 379; 1993). Their conclusion is that a smaller proportion than previously thought of the substance of a neutron star is embodied in the outer crust; within that is nuclear matter proper.

H. Heiselberg from the Nils Bohr Institute, together with Pethick and E.F. Staubo from Illinois, have now taken the argument a step further, defining the condition if drops of quark matter are to form within a matrix of nuclear matter (Phys. Rev. Lett. 70, 1355-9; 8 March 1993). They say this picture of the matter inside a neutron star could have important observational consequences; the deformation of the non-spherical oddly shaped drops might be linked to occasional sudden acceleration of the rotation of neutron stars. But, as for the past twenty years, the nuclear data were not good enough to be sure, and are not nearly good enough yet to be sure.

John Maddox 